\title{
Foil-to-Foil System Integration Through Capillary Self-Alignment Directed by Laser Patterning
}

\author{
Gari Arutinov, Massimo Mastrangeli, Edsger C. P. Smits, Gert van Heck, \\ Jaap M. J. den Toonder, and Andreas Dietzel
}

\begin{abstract}
This paper introduces a new integration technology for cost-effective high-precision mechanical and electrical integration of mesoscopic functional foil components onto foil substrates. The foil-to-foil assembly process is based on topological surface structuring via laser patterning that enables accurate capillaritydriven self-alignment of foil dies. The concurrent establishment of high-yield electrical interconnections is obtained through conductive adhesives. The foil surface energy controls the acceptance window of initial offsets for optimal self-alignment performance. The proposed topological patterning and system design enable alignment accuracies for centimeter-sized foil dies as high as $15 \mu \mathrm{m}$, barely influenced by the evaporation of the assembly liquid and curing of the conductive paste. Full foil-to-foil system integration is demonstrated through the electrically functional assembly of an array of Au-sputtered capacitive humidity sensors onto a patterned base foil circuitry.

[2014-0008]
\end{abstract}

Index Terms-Capillarity, fluidics, foil-to-foil integration, self-alignment, self-assembly.

\section{INTRODUCTION}

$\mathbf{T}$ HE DEMAND for smart and multi-functional flexible devices such as sensors-in-foil [1], [2] has significantly increased in recent years [3]. The rise of lowcost, low-complexity and highly-reliable integrated systems [4] capable of sensing and interacting with their environment [5] requires new technological developments. To address this need, cost- and time-effective integration of components

Manuscript received January 13, 2014; revised March 11, 2014; accepted April 23, 2014. This work was supported by the Marie Curie ITN FlexSmell Project under Grant 238454. The work of M. Mastrangeli was supported in part by the Nano-Tera.Ch Swiss Research Initiative through the SelfSys Project, and in part by the Interuniversity Attraction Poles Programme under Grant IAP 7/38 MicroMAST through the Belgian Science Policy Office. Subject Editor L. Spangler.

G. Arutinov, E. C. P. Smits, and G. van Heck are with the Holst Centre, Netherlands Organisation for Applied Scientific Research, Eindhoven 5656 AE, The Netherlands (e-mail: gari.arutinov@gmail.com; edsger.smits@tno.nl; gert.vanheck@tno.nl).

M. Mastrangeli was with the Microsystems Laboratory, École Polytechnique Fédérale de Lausanne, Lausanne 1015, Switzerland. He is now with the Department of Bio, Electro, and Mechanical Systems, École Polytechnique, Université Libre de Bruxelles, Brussel 1050, Belgium (e-mail: massimo.mastrangeli@ulb.ac.be).

J. M. J. den Toonder is with the Department of Mechanical Engineering, Eindhoven University of Technology, Eindhoven 5600 MB, The Netherlands (e-mail: j.m.j.d.toonder@tue.nl).

A. Dietzel is with the Institute of Microtechnology, Technical University of Braunschweig, Braunschweig 38124, Germany (e-mail: a.dietzel@tubraunschweig.de).

Color versions of one or more of the figures in this paper are available online at http://ieeexplore.ieee.org.

Digital Object Identifier 10.1109/JMEMS.2014.2321013 of complementary functionality-e.g. logic, memory, power, transceiver and sensor units- into standalone devices on non-standard substrates such as plastic foils [6], [7] is imperative. Fabrication processes of many such components are often mutually incompatible and do not allow for monolithic integration [8]. Therefore, heterogeneous or hybrid integration onto carrier substrates is regarded as a promising alternative. Hybrid integration additionally allows an increase in the overall fabrication yield and shortened design optimization cycles, since it allows for separately testing the functionality of the components before their integration [9].

For the past few decades, high-precision robotic pick-andplace has been established as the technique of choice for system integration in electronic manufacturing [10] thanks to its unmatched programmable dexterity in handling components of arbitrary shape [11]. However, pick-and-place is inherently serial and currently faces an accuracy versus throughput tradeoff in the assembly of (sub-)millimetric components [12]. This has motivated the development of a vast class of alternative or complementary techniques for heterogeneous system integration based on self-assembly [13]. Self-assembly has been implemented in many applications [14], [15] using differing principles and physical interactions, such as shape recognition [8], [16], as well as electrostatic [17], magnetic [18], and capillary forces [19], [20]. Within this context, capillary selfalignment in particular is being thoroughly investigated as it can achieve registration accuracy for millimetric [13], [21] and micrometric [22] components limited only by lithographical or dicing tolerances. It can moreover leverage and complement flip-chip assembly to overcome its performance limitations [23].

A typical procedure for the integration of functional components onto target carrier substrates includes two major steps: 1) accurate registration of the components onto pre-processed binding sites, and 2) establishment of electrical interconnections between the components and the electrodes embedded in the sites. As compared to robotic flip-chip assembly, in capillary self-alignment both steps are mediated by the deposition and confinement on the binding sites of a specified amount of interposing liquid [24]. Liquid confinement can be enforced through either topographical [25] and/or chemical [24] surface structuring. Earlier works by Smith [26], Morris [27], and Chung [20] and Taprogge [28] showed how the parallel formation of working electrical and mechanical interconnections can be included in fluidic self-assembly processes. Xiong used 
electroplating to form vertical electrical links from substrate to microcomponents after their capillary self-assembly [29]; whereas Mastrangeli [24], [30] demonstrated how the hydrostatic pressure exerted by residual liquid menisci on capillary self-aligned components can be successfully exploited to establish solder-based interconnections upon thermal reflow, as an alternative to thermo-compression bonding [21]. The use of capillary self-alignment for low-cost and time-efficient assembly of centimetric functional foil dies onto silicon and flexible plastic substrates was demonstrated in our previous work [13]. Further improvement in assembly throughput could derive from the optimization of the technique for large-scale roll-to-roll manufacturing [6], [7].

To address this technological challenge, in this paper we introduce a novel technology that enables the high-precision mechanical and electrical integration of mesoscopic functional foil components onto foil substrates. The foil-to-foil assembly process is based on topological surface structuring via laser patterning. It achieves facedown capillary self-alignment of centimetric foil dies over matching target positions as accurate as $15 \mu \mathrm{m}$ and concurrent realization of high-yield electrical interconnections with base foil carriers through conductive adhesives. This enables, for instance, the integration of mesoscopic foil interposers embedding dense arrays of miniaturized components, such as bare Si chips and bare die LED's [31].

Unlike silicon chips, foil-based components can be patterned and diced by programmable, fully dry and nonlithographic laser ablation without additional surface chemical functionalization [13]. In this work, laser sources are used to pattern as well as to compartmentalize both donor and acceptor foils in a single process step. Römer et al. earlier developed a laser micromachining process to create sharp-edged receptor sites for capillary self-alignment [32]. Their study mostly focused on the physico-chemical mechanism shaping trenches for binding sites upon picosecond laser ablation rather than on its influence on capillary self-alignment performance. Besides, submillimetric SU-8 dies were used thereby as assembly vehicles, which require high-precision robotic equipment for die pre-alignment with respect to the corresponding binding sites [13]. Here we investigate the performance of capillary self-alignment onto laser-patterned substrates of mesoscopic foil dies, which, given the dimensions of the vehicles, is more tolerant against initial die misalignment and, by the same token, does not necessarily require any high-precision prealignment system. We demonstrate that foil patterning with nano- and picosecond laser sources with similar wavelength and spot size yield no relevant difference. Thus, both can be equally well employed for structuring and dicing foil dies and carrier substrates.

For each foil die, our capillary self-alignment process proceeds as follows [Fig. 1]:

a) An outer trench is laser-scribed onto the carrier to create a binding site matching the shape and size of the foil die, and an inner circular trench is laser-scribed in the center of the binding site.

b) A liquid droplet is dispensed within the inner trench, where it remains confined with a predictable profile.

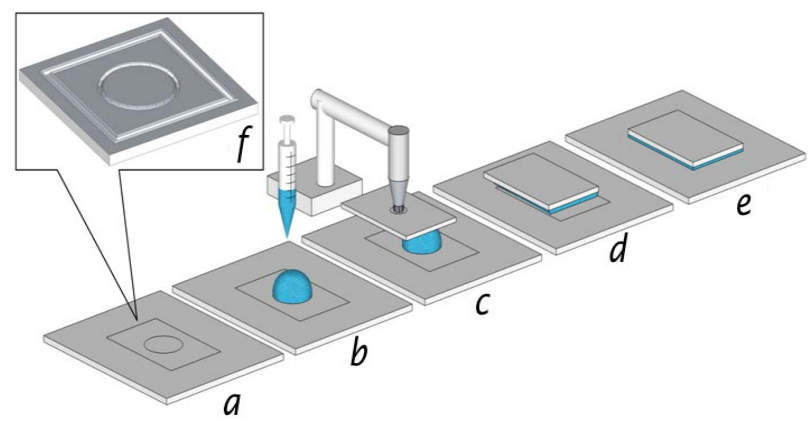

Fig. 1. Sketch of the process steps for laser-structure supported foil-to-foil capillary self-alignment: (a) laser patterning of the binding site on the carrier substrate, including the inner confinement ring, (b) liquid dispensing within the confinement ring, (c) coarse pre-alignment of foil die, (d) formation of the liquid meniscus, and (e) constrained self-alignment of the foil die on the corresponding binding site.

c) A foil die is coarsely aligned with the binding site and brought in contact with the liquid droplet by means of a micro positioner.

d) As a consequence, the liquid wets the foil die forming a meniscus.

e) Upon release of the foil die, liquid meniscus relaxation, constrained by edge confinement and geometrical shape matching, self-aligns the foil die to the corresponding binding site.

Upon die self-alignment, electrical interconnections to the carrier electrodes mediated by conductive adhesives are established to complete the foil-to-foil integration process.

\section{Materials AND Methods}

Foil dies and carrier substrates were fabricated from transparent $125-\mu \mathrm{m}$-thick Polyethylene Naphthalate (PEN) sheets (Teonex ${ }^{\circledR}$ Q65FA, DuPont). Nano- (Coherent AVIO, $355 \mathrm{~nm}, 25 \mathrm{~ns}$ ) and picosecond (Coherent Talisker, $355 \mathrm{~nm}$, 15 ps) Nd:YAG laser sources were used for patterning and dicing foil dies and carrier substrates. The dies were handled through a homebuilt micropositioning stage equipped with integrated vacuum tweezers (SMD-VAC-HP, Vacuum Industries, Inc.). A stylus profiler (DektakXT, Brukker) was used for profilometric analysis of laser-scribed trenches. A positioning base stage (XYZ 500 TIS, Quater Research and Development) providing $10 \mu \mathrm{m}$-resolved displacements along three axes was used to pre-align the dies. Oxygen plasma treatment at $400 \mathrm{~W}$ for $1 \mathrm{~min}$ was used to enhance wettability of PEN foils. Contact angles were measure by the static sessile drop method (EasyDrop, KRÜSS). High-speed camera stage (Redlake HS-3) and image recognition software were used to track and analyze the process dynamics. A $250 \mu \mathrm{m}$-thick PEN film with gold-sputtered interdigitated capacitive humidity sensors featuring $1 \mathrm{~mm}$-pitch electrodes (0.7 mm-wide interconnect lines with a $0.3 \mathrm{~mm}$-wide gap) and a $125 \mu \mathrm{m}$-thick PEN base circuitry foil of an RFID tag consisting of screen printed silver tracks (5025, DuPont) with a row of matching electrodes were used for foil-tofoil integration experiments. The sensor foils and carrier substrates were patterned and diced with the nanosecond laser. 


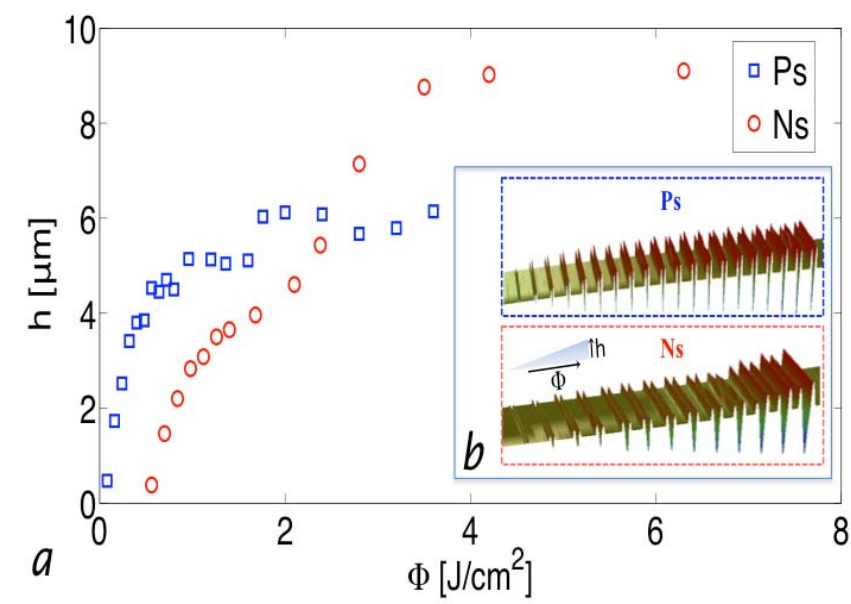

Fig. 2. (a) Height of humps recast around laser-scribed lines on PEN foil as function of pico- (ps) and nanosecond (ns) integral laser fluence (Nd:YAG lasers, $355 \mathrm{~nm}$, spot size: $45 \mu \mathrm{m}$ ), and (b) profilometric analysis of corresponding laser-scribed lines.

An epoxy-based Ag-filled isotropic conductive adhesive (ICA) (CE-3103 WLV, Henkel) was stencil-printed on silver tracks to interconnect matching electrical contacts of the foils. Functional foil dies were handled with manual vacuum tweezers (Thorlabs) for integration experiments. ICA was cured at $120{ }^{\circ} \mathrm{C}$ for $15 \mathrm{~min}$ in a conventional oven. The capacitance values of Au-sputtered sensor array foils were measured through an LCR impedance analyzer (Agilent, E4980A).

\section{RESULTS AND DisCUSSIONS}

\section{A. Foil-to-Foil Self-Alignment}

1) Laser Patterning: It is generally accepted that laser ablation of plastic surfaces can proceed by both photothermal and photochemical mechanisms. Dominance of either mechanism depends on laser fluence and wavelength [33]. It was shown that using 355-nm nanosecond Nd:YAG laser, the ablation of PEN by sub- and near-threshold fluences generates humps by recasting of ablated material around the scribed region [34]. Similar humps were obtained for polyimide foils using a 343-nm picosecond laser [32]. To determine the relevance of the laser pulse length on the formation of microhumps on PEN foil, a comparative study of the ablation of PEN foils using pico- and nanosecond laser sources with equal wavelength (355 $\mathrm{nm})$ and spot size $(45 \mu \mathrm{m})$ was performed. We scribed a set of lines on $125-\mu \mathrm{m}$-thick Polyethylene Naphthalate (PEN) sheets using a range of fluences. Profilometric analysis evidenced the microhumps around laser-scribed lines and, for both types of lasers, a similar correlation between the height of the humps and corresponding laser fluence was obtained [Fig. 2]. The height of the microhumps is roughly linear with laser fluence for low fluence values, and eventually saturates upon further increase of laser fluence. Notably, hump-decorated sites resemble recessed sites in the way they are surrounded by perimetric sidewalls [24]. The microhumps 1) cause strong pinning of the triple contact line along the entire edge of the sites, and 2) provide an additional barrier against liquid overflow [24]. A significant enhancement of

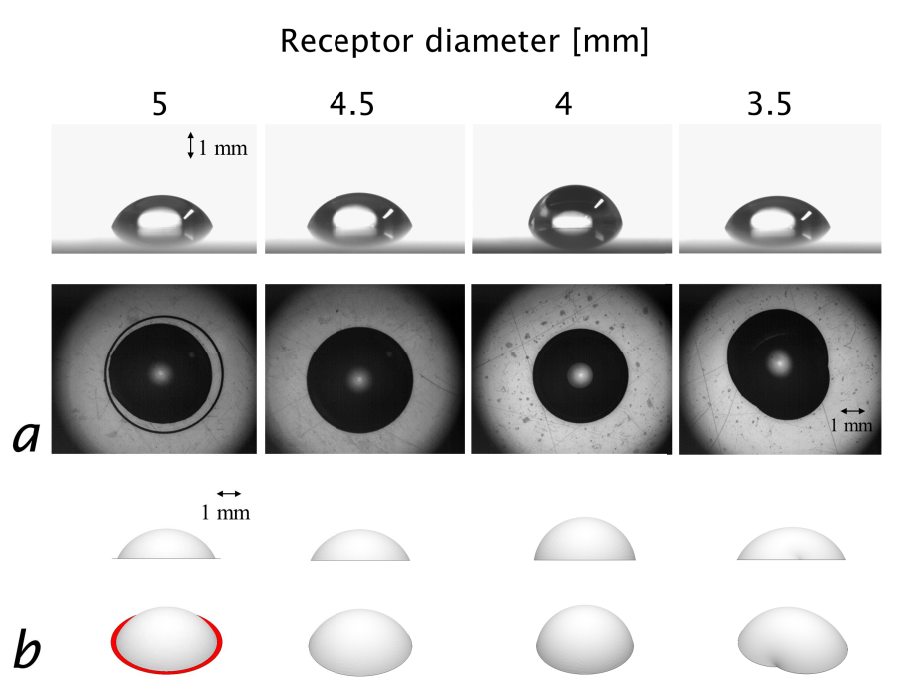

Fig. 3. Side and top view images of a $12.5 \mu 1$ water droplet on a set of circular receptor sites of varying diameter (indicated above the images) with laserscribed outline. (a) Optical pictures, (b) Surface Evolver [36] simulations. Dewetting and overspreading are apparent on relatively large and small circular sites, respectively.

liquid confinement within these sites as compared to sites with flat, straight edges could thus be consistently expected (refer to Fig. 6 below).

Controlled and repeatable performance of capillary self-alignment requires the dispensing of well-defined amounts of assembly liquid on the binding sites. We used site-related water volumes to result in a uniform, $125 \mu$ m-thick layer after spreading over the whole site surface upon full die alignment, consistent with our prior optimization [13]. Given the non-null contact angle $\left(65^{\circ} \pm 3^{\circ}\right.$ of water on native PEN, coverage of unpatterned sites by droplets of optimal volume is only partial and non-reproducible [23]. Conversely, droplets deposited within the inner circular trenches at the center of the sites are reproducibly localized and assume a predictable profile. The diameter of the laser-scribed ring was optimized to achieve conformal confinement of the optimal droplets, i.e., full coverage of the inner circular area. Fig. 3 shows that, for a $12.5 \mu \mathrm{l}$ water droplet, the optimal diameter of the confinement ring is $4.5 \mathrm{~mm}$. Such tight edge confinement additionally yields an immediate water overflow beyond the circular trench [35] and a more reproducible initial spreading of the water meniscus across the binding site upon landing of the foil die.

To investigate the efficacy of the proposed laser-based topological patterning, sets of nominally identical $10 \times 10 \mathrm{~mm}^{2}$ foil dies were self-aligned onto matching binding sites with optimal inner rings. The sites were patterned on dummy carrier substrates by both lasers with fluences above their saturation values [Fig. 4]. The dies were released from an initial offset of $1 \mathrm{~mm}$ (i.e. $10 \%$ of their side length), and the final dieto-site registration was quantified by analyzing the matching of marker structures engraved on the foil die and the carrier substrate.

Shortly after water dispensing [Fig. 4(b) and Fig. 4(e)], the foil dies were coarsely superposed onto the binding sites 


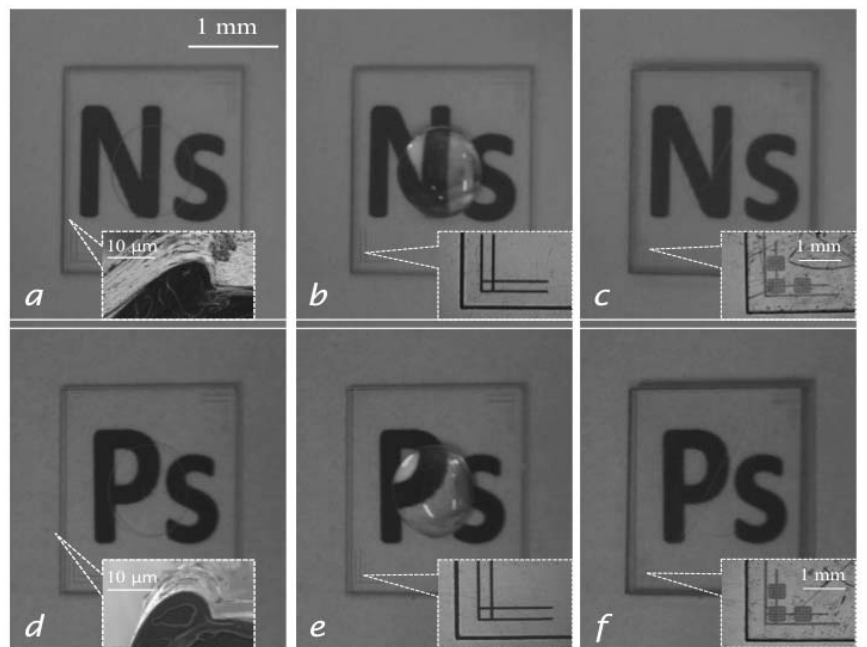

Fig. 4. Optical images of capillary self-alignment of $10 \times 10 \mathrm{~mm}^{2}$ foil dies onto carrier foils patterned by nano- (a-c) and picosecond (d-f) lasers. High precision alignment was registered for both cases despite the difference in shape of the ablated groove [insets in (a) and (d), respectively].

through a micropositioner while held a few millimeters above the droplets. Thanks to prior knowledge of the droplet height, the foil dies could be lowered to precisely contact the top of the droplets and form the capillary bridges. The procedure ensures accurate control over pre-alignment and release of the foil dies. A self-alignment yield of $95 \%$ and an alignment accuracy of $15 \pm 6 \mu \mathrm{m}$ were obtained from 20 experiments performed for each laser patterning type [Fig. 4(c) and 4(f)]. No performance dependence on the type of laser scribing was noticed. This result can be explained by the similarity of the inner sidewalls of the microhumps produced by both types of lasers, despite the different shape of the grooves [see insets of Fig. 4(a) and 4(d)]. Thus both nano- and picosecond lasers can be equivalently employed for foil-to-foil capillary selfalignment. In the following, the nanosecond laser, being more accessible, was chosen as the primary laser source.

2) Initial Misalignment: A systematic study of the impact of the initial die offset - known to influence the dynamics and final registration accuracy of capillary self-alignment [13], [37] - was conducted for our process. A $10 \times 10 \mathrm{~mm}^{2}$ foil die was released five times from each of the chosen initial offsets. The results point to the presence of an optimal offset window, i.e., a discernible range of initial offsets for which foil dies self-align reproducibly and with high accuracy [Fig. 5(a)].

Moreover, the self-alignment process yields a binary result. Depending on its initial offset, a released foil either immediately fails to align and in this case sticks at its release position due to water overspreading - or it successfully self-aligns with an accuracy of 10 to $15 \mu \mathrm{m}$ [Fig. 6]. Indeed, and contrary to the all-chemical patterning of foil carriers [13], [37], liquid overspreading beyond laser-structured trenches is nonreversible, i.e., the liquid cannot retract back onto the binding site. It is important to note that the use of the same laser system for patterning and dicing both carrier substrates and foil dies clears instrumental tolerances (estimated to be $30 \mu \mathrm{m}$ ) and facilitates self-alignment with the demonstrated alignment accuracy.
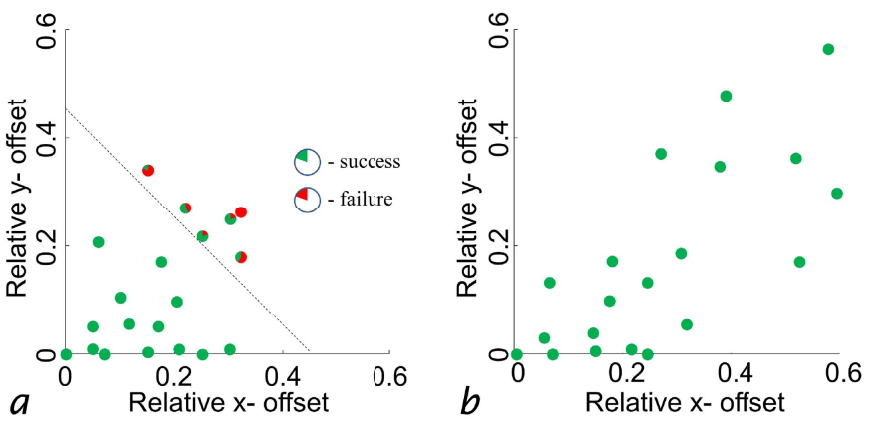

Fig. 5. Self-assembly yield of (a) native and (b) $\mathrm{O}_{2}$-plasma treated $10 \times 10 \mathrm{~mm}^{2}$ PEN foil die as a function of initial release offsets (normalized to die side length). In both cases foil dies were self-aligned onto a patterned native PEN carrier foil. For each initial offset value, five experiments were performed, and corresponding success rates are illustrated by pie charts.

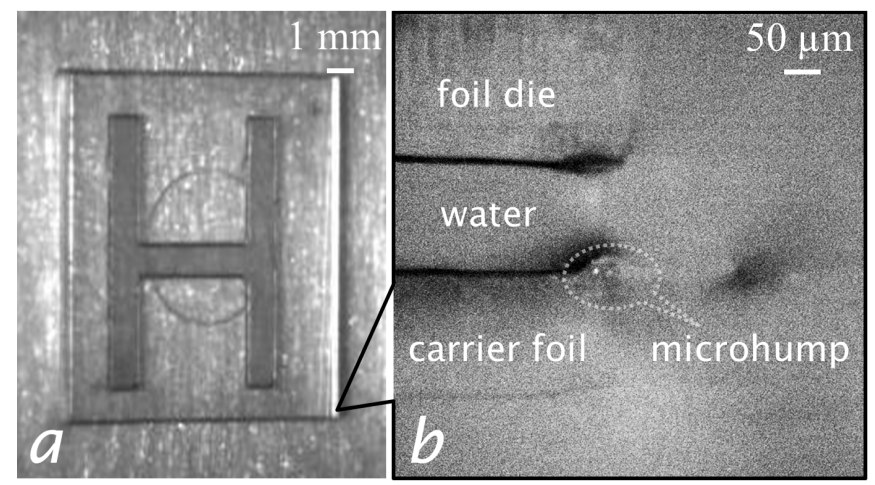

Fig. 6. Side (a) and top (b) view images of $10 \times 10 \mathrm{~mm}^{2}$ foil die self-aligned onto patterned carrier foil. The laser-induced microhumps enhance liquid confinement within the receptor site, as expected from recessed sites [24].

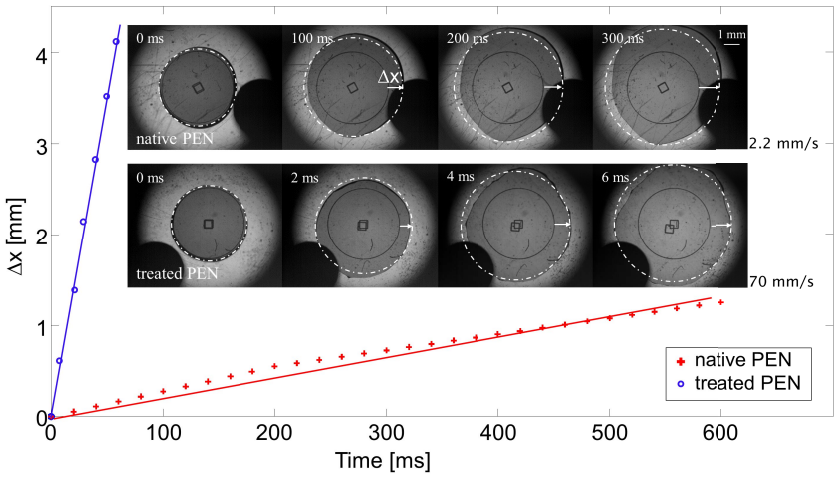

Fig. 7. Spreading dynamics of the water meniscus initially confined within a circular trench $\left(4.5 \mathrm{~mm}\right.$ in diameter) over native and $\mathrm{O}_{2}$-plasma treated PEN foil substrates upon release of a $10 \times 10 \mathrm{~mm}^{2}$ bare PEN foil die. Slightly asymmetric spreading of water was averaged (see circles on inset frames).

3) Surface Energies: The energy of the surfaces constraining the liquid meniscus impacts the dynamics of capillary self-alignment [37]. Foil-to-foil capillary self-alignment is characterized by significantly longer assembly times with respect to foil-to-silicon/silicon-to-foil assembly [38]. Given the same assembly liquid (water), this can be explained by the lower surface energy of and consequent slower liquid spreading on native PEN foils relative to native silicon dioxide [39]. 


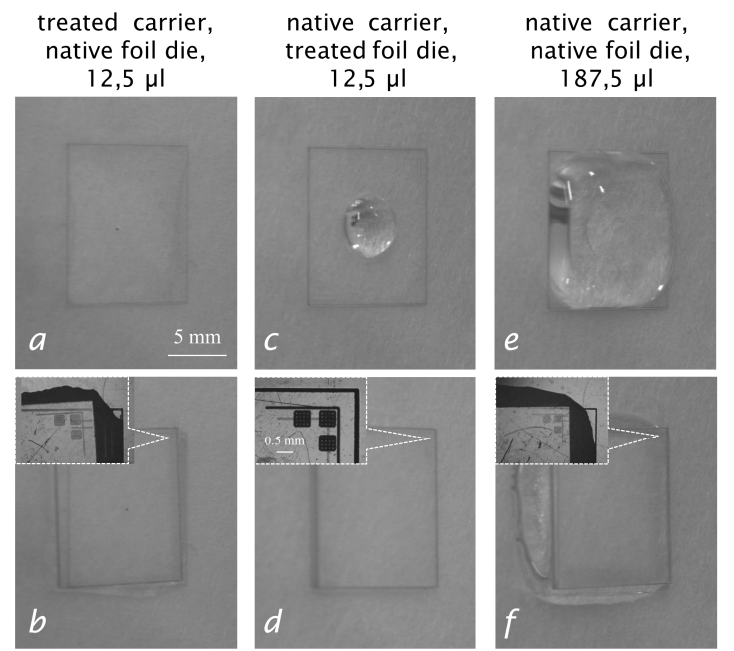

Fig. 8. Optical images of (a) $12.5 \mu \mathrm{l}$ of water spread over an $\mathrm{O}_{2}$-plasma treated carrier foil and (b) corresponding failed alignment of a matching native foil die due to water overspreading; (c) $12.5 \mu \mathrm{l}$ of water pre-confined within the inner circular trench on a native carrier foil and (d) corresponding correct alignment of a matching $\mathrm{O}_{2}$-plasma treated foil die; (e) $187.5 \mu \mathrm{l}$ of water spread over a native carrier foil and (f) corresponding failed alignment of a matching native foil die due to water overspreading. Self-alignment of native foil die onto native carrier foil is shown on Fig 4(a-c). In all cases the carrier foils consisted of a $10 \times 10 \mathrm{~mm}^{2}$ binding site with a laser-scribed, $4.5 \mathrm{~mm}$ in diameter inner circular trench.

Three sets of self-alignment experiments were performed with $10 \times 10 \mathrm{~mm}^{2}$ foil dies to assess the spreading dynamics of the water menisci across the sites [Fig. 7]. First, as standard case, untreated foil dies were self-aligned onto untreated carrier substrates [Fig. 4(a-c)]. Two more sets of experiments involved untreated foil dies onto $\mathrm{O}_{2}$-plasma treated, hydrophilic base foils (water CA: $8^{\circ} \pm 2^{\circ}$ ) and, in reverse modality, equally treated foil dies onto hydrophobic carrier substrates. The results demonstrated a significant increase (over 30 times) of the water spreading velocity for the case of either hydrophilic dies or hydrophilic substrates relative to the standard case.

Whereas water spreading was accelerated, capillary self-alignment experiments on hydrophilic carrier foils systematically failed for all initial offsets ranging from 0.15 to $6 \mathrm{~mm}$. This is attributed to the inability of hydrophilic laser-scribed trenches to hold the water inside the sites [Fig. 8(a) and 8(b)]. Conversely, no overspreading was observed for hydrophilic foil dies, which self-aligned onto native carrier foils with $17 \pm 4 \mu \mathrm{m}$ accuracy and $100 \%$ yield out of 25 experiments [Fig. 8(c) and 8(d)]. In addition, for this modality a noticeably larger optimal window of initial offsets was observed [Fig. 5(b)]. Particularly, foil dies released from initial offsets equal to $60 \%$ of their lateral dimension could still be self-aligned with the best accuracy.

Additionally, self-alignment was attempted with native PEN surfaces for both foil die and carrier in the presence of an amount of water $(187.5 \mu \mathrm{l})$ covering $97 \%$ of the $10 \times 10 \mathrm{~mm}^{2}$ binding site from the outset [Fig. 8(e)]. Only dewetting from corners prevented full site coating [24], [40], [41]. In this setting, overspreading of the excessive amount of water over the microhumps and consequent alignment failure were

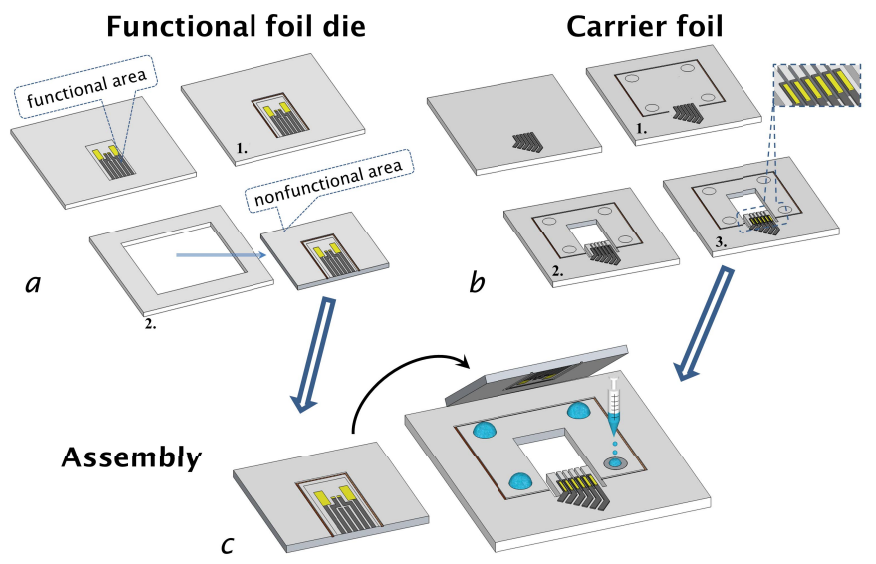

Fig. 9. Process steps for foil-to-foil electro-mechanical integration. (a) Patterning and dicing of the functional foil die: 1) laser-scribing to isolate functional and non-functional areas; 2) dicing of the functional foil. (b) Patterning of the carrier foil: 1) laser-scribing of a binding site of the same shape and size as the functional foil die; and patterning of the circular trenches in the corners of the binding site; 2) laser-ablation of the access window; 3) stencil-printing of thin layer of ICA paste on the electrodes. (c) Dispensing of water onto the circular receptor sites on the base foil, and facedown capillary self-alignment of the foil die followed by curing of the ICA paste.

consistently registered [Fig. 8(f)]. Such failure could be overcome by increasing the hydrophobicity of the receptor sites, as suggested in [42].

\section{B. Foil-to-Foil Integration}

To complete the integration, electrical interconnections need to be established. In this respect, liquids typically used as an assembly medium for capillary self-alignment (for instance, water) are isotropically conductive and therefore incompatible with conductive materials required for electrical interconnections, such as isotropic conductive adhesives (ICA). Recently Landesberger [43] suggested the use of an anisotropic conductive adhesive (ACA) as both an assembly medium for capillary self-alignment and as a conductive material to electrically connect thin silicon chips onto polyamide foil substrates. However, no functionality of the integrated system was shown.

As an alternative approach, we employ a technique whereby foil-to-foil self-alignment and interconnection are performed sequentially and without interference by means of laser-enabled separation of (electrically) functional and non-functional areas. The process is defined as follows [Fig. 9]:

a) A foil die containing specific functionality, such as a printed sensor, is laser-patterned and diced:

1. A trench is laser-scribed around the functional area to isolate it from the rest of the surface of the carrier foil;

2. The functional foil die with separated (functional and nonfunctional) areas is laser-diced.

b) A base carrier foil with printed electrodes is patterned:

1. A trench is laser-scribed to create a binding site matching the shape and size of the trench 
separating the areas on the foil die, and four circular water-confining trenches are laser-scribed near the corners of the binding site. The latter allows the deposition of identical droplets over the surface, ensuring better control over pre-alignment and release of the foil dies;

2. Optionally, a window is laser-ablated to provide access to the functional area of the foil die after its facedown integration onto the base foil;

3. A layer of isotropic conductive adhesive (ICA) paste $(25 \mu \mathrm{m})$ thinner than the height of the water menisci $(125 \mu \mathrm{m})$ is stencil-printed on the electrodes of the base foil to provide an electrical connection with the matching metal tracks on the functional foil die.

c) Water is dispensed onto the circular receptor sites on the base foil, and facedown self-alignment of the functional die is performed. Upon evaporation of the water, the metal tracks on the foil die touch the ICA paste film. The ICA is then cured to establish foil-to-foil interconnection.

Demonstration of the integration process was performed through a $35 \times 30 \mathrm{~mm}^{2}$ foil die embedding a $15 \times 15 \mathrm{~mm}^{2}$ $\mathrm{Au}$-sputtered interdigitated electrode (IDE) array that can be used as capacitive humidity sensor [7]. It features standardized flex flat cable interconnections with $1 \mathrm{~mm}$-pitch electrodes. The die was integrated onto $125 \mu$ m-thick RFID tag base foils featuring matching screen-printed silver tracks [Fig. 10(a)]. Given the $0.7 \mathrm{~mm}$-wide electrode lines with $0.3 \mathrm{~mm}$-wide gap, component registration accuracies on the order of $50 \mu \mathrm{m}$ or less are necessary. The functional foil die was coarsely pre-aligned to the corresponding binding site [Fig. 10(b)] and then released onto the four droplets (see supplementary material for related self-alignment video). As reported in Sect. III.A.2, treated foil dies show a wider optimal offset window compared to native foil dies [Fig. 5]. Still, the acceptance window's width available for untreated foil dies [Fig. 5(a)] was sufficient for our application, so that manual self-alignment and subsequent integration of the functional foil die could be directed only by topological laser patterning, thus avoiding additional surface treatment. Alignment accuracy better than $15 \mu \mathrm{m}$ was obtained [Fig. 10(c) and 10(d)] with the interconnect lines precisely superposed [Fig. 10(e)]. The same assembly precision was observed after bonding of the foil die by curing the ICA paste [Fig. 10(f) and 10(g)]. Thus the final integration step, including the evaporation of the assembly liquid and curing of the conductive paste, did not impede the high registration accuracy achieved via prior capillary self-alignment. The capacitance values of an array of interdigitated electrode sensors were measured before and after the integration with an LCR impedance analyzer. The sensors have differing geometries resulting in different capacitances. For all six sensors the measured capacitance values after bonding were in a good agreement with the initial values [Fig. 10(h)]. A small, systematic change in capacitance was observed due to the parasitic wiring onto the base foil. An identical IDE array-previously integrated via standard pick-and-place assembly [7], and showing equal

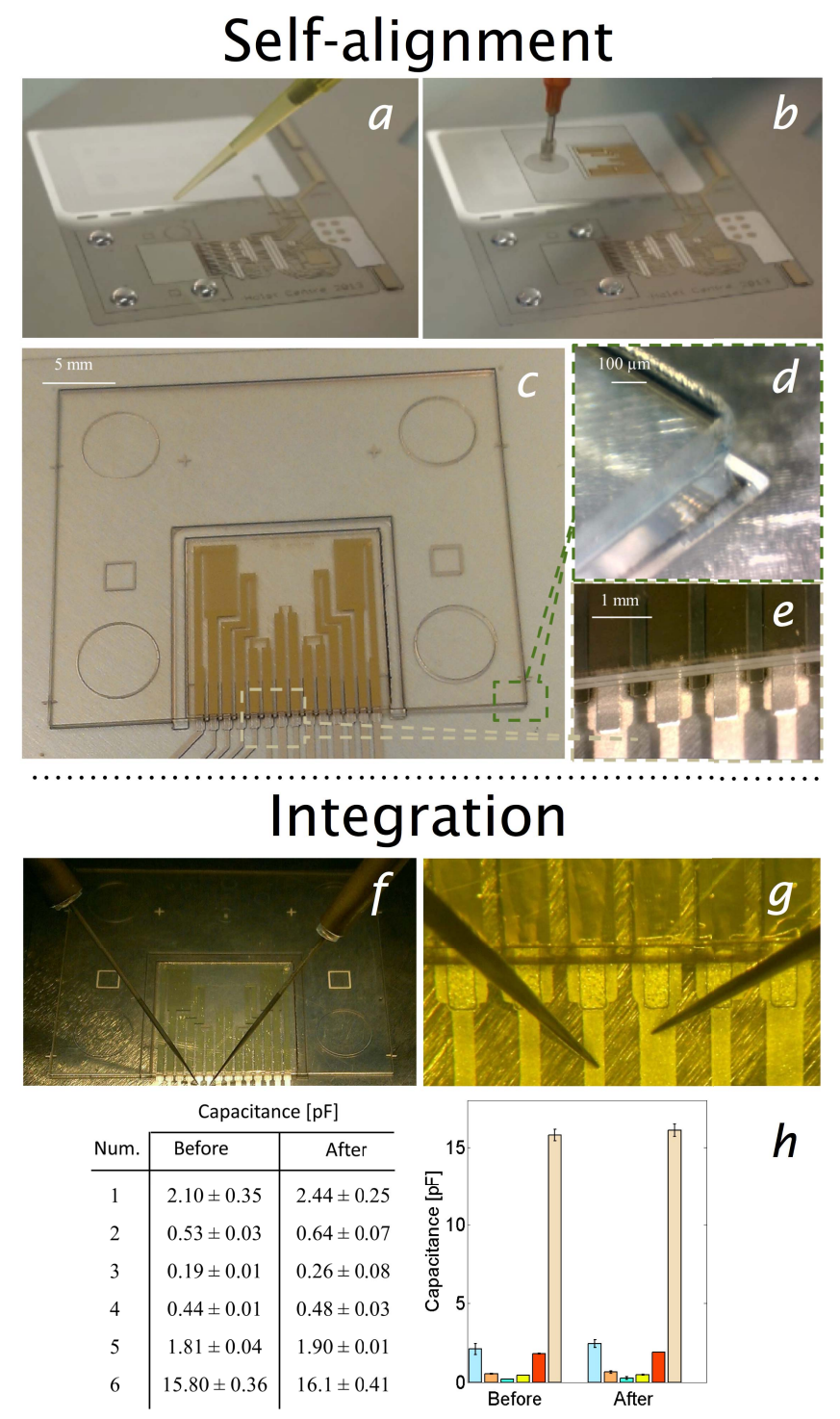

Fig. 10. Optical images of (a) $180 \mu \mathrm{l}$ of water confined within four $6 \mathrm{~mm}$ in diameter circular acceptor sites laser-scribed on a base foil. (b) A coarsely pre-aligned $35 \times 30 \mathrm{~mm}^{2}$ functional foil die with Au-sputtered $15 \times 15 \mathrm{~mm}^{2}$ interdigitated capacitive humidity sensor array. (c) The sensor foil after selfalignment onto a patterned base foil with printed circuitry. (d) Closeup top view of the $0.7 \mathrm{~mm}$ wide interconnect lines with a $0.3 \mathrm{~mm}$ wide gap, and (e) side view of liquid meniscus sandwiched between self-aligned foil die and corresponding binding site on a carrier foil. (f) The sensor foil after the integration onto a carrier substrate. (g) Close-up top view featuring well aligned metal tracks connected to the matching silver electrodes on the base foil. (h) Comparison between the measured capacitance values before and after integration for the whole array of capacitive sensors.

capacitive values as in the present case both before and after bonding — was successfully used for capacitive humidity sensing [7].

\section{CONCLUSION}

We have introduced a new technology for high-precision mechanical and electrical integration of mesoscopic functional foil systems. The foil-to-foil assembly exploits laser-based topological surface structuring to achieve both accurate foil die capillary self-alignment and electrical interconnection of the foil die to base foil carriers through conductive adhesives. 
We have identified an acceptance window of initial die offsets allowing centimeter-sized dies to align with accuracy limited only by patterning tolerances. We have also shown how such window as well as liquid spreading and concurrent die self-alignment dynamics can respectively be widened and accelerated by tailoring the surface energies of the bounding surfaces. Water evaporation and curing of the conductive paste during bonding barely influence the high registration accuracy achieved by prior capillary self-alignment. Electromechanical full system assembly was demonstrated through the integration of functional foils connecting an array of Au-sputtered capacitive humidity sensors onto patterned circuitry foils. The technology enables the efficient, low-cost and large-scale integration of functional systems-infoil applications.

\section{ACKNOWLEDGMENTS}

The authors would like to thank Roel Kusters for conductive adhesive deposition and Merijn Giesbers for providing highresolution visualizing setup.

\section{REFERENCES}

[1] J. Courbat, D. Briand, L. Yue, S. Raible, and N. F. de Rooij, "Dropcoated metal-oxide gas sensor on polyimide foil with reduced power consumption for wireless applications," Sens. Actuators B, Chem., vol. 161, no. 1, pp. 862-868, Jan. 2012.

[2] U. Altenberend et al., "Towards fully printed capacitive gas sensors on flexible PET substrates based on Ag interdigitated transducers with increased stability," Sens. Actuators B, Chem., vol. 187, pp. 280-287, Oct. 2013.

[3] D. Briand, A. Oprea, J. Courbat, and N. Bârsan, "Making environmental sensors on plastic foil," Mater. Today, vol. 14, no. 9, pp. 416-423, Sep. 2011

[4] L. Atzori, A. Iera, and G. Morabito, "The internet of things: A survey," Comput. Netw., vol. 54, no. 15, pp. 2787-2805, Oct. 2010.

[5] D. Briand, F. Molina-Lopez, A. V. Quintero, G. Mattana, and N. F. de Rooij, "Printed sensors on smart RFID labels for logistics," in Proc. Int. IEEE New Circuits Syst. Conf., Jun. 2012, pp. $449-452$.

[6] R. A. Potyrailo, A. Burns, C. Surman, D. J. Lee, and E. McGinniss, "Multivariable passive RFID vapor sensors: Roll-to-roll fabrication on a flexible substrate," Analyst, vol. 137, no. 12, pp. 2777-2781, Feb. 2012.

[7] A. Vásquez Quintero et al., "Foil-to-foil lamination and electrical interconnection of printed components on flexible substrates," Microelectron. Eng., vol. 110, pp. 52-58, Oct. 2013.

[8] S. A. Stauth and B. A. Parviz, "Self-assembled single-crystal silicon circuits on plastic," Proc. Nat. Acad. Sci. United States Amer, vol. 103 , no. 38, pp. 13922-13927, Sep. 2006.

[9] R. S. Patti, "Three-dimensional integrated circuits and the future of system-on-chip designs," Proc. IEEE, vol. 94, no. 6, pp. 1214-1224, Jun. 2006.

[10] C. J. Morris, S. A. Stauth, and B. A. Parviz, "Self-assembly for microscale and nanoscale packaging: Steps toward self-packaging," IEEE Trans. Adv. Packag., vol. 28, no. 4, pp. 600-611, Nov. 2005.

[11] Y. Zhang, B. K. Chen, X. Liu, and Y. Sun, "Autonomous robotic pick-and-place of microobjects," IEEE Trans. Robot., vol. 26, no. 1, pp. 200-207, Feb. 2010.

[12] M. Gauthier and S. Régnier, Eds., Robotic Micro-Assembly. Hoboken, NJ, USA: Wiley, 2011.

[13] G. Arutinov et al., "Capillary self-alignment of mesoscopic foil components for sensor-systems-in-foil," J. Micromech. Microeng., vol. 22, no. 11, pp. 115022-115031, Nov. 2012.

[14] M. Mastrangeli, S. Abbasi, C. Varel, C. Van Hoof, J. P. Celis, and K. F. Bohringer, "Self-assembly from milli-to nanoscales: Methods and applications," J. Micromech. Microeng., vol. 19, no. 8, pp. 083001-083037, Aug. 2009.

[15] T. G. Leong, A. M. Zarafshar, and D. H. Gracias, "Three-dimensional fabrication at small size scales," Small, vol. 6, no. 7, pp. 792-806, Mar. 2010.
[16] W. Zheng, J. Chung, and H. O. Jacobs, "Fluidic heterogeneous microsystems assembly and packaging," J. Microelectromech. Syst., vol. 15, no. 4, pp. 864-870, Aug. 2006.

[17] J. Dalin, J. Wilde, A. Zulfiqar, P. Lazarou, A. Synodinos, and N. Aspragathos, "Electrostatic attraction and surface-tension-driven forces for accurate self-assembly of microparts," Microelectron. Eng., vol. 87, no. 2, pp. 159-162, Feb. 2010.

[18] S. B. Shetye, I. Eskinazi, and D. P. Arnold, "Magnetic self-assembly of millimeter-scale components with angular orientation," J. Microelectromech. Syst., vol. 19, no. 3, pp. 599-609, Jun. 2010.

[19] R. J. Knuesel and H. O. Jacobs, "Self-assembly of microscopic chiplets at a liquid-liquid-solid interface forming a flexible segmented monocrystalline solar cell," Proc. Nat. Acad. Sci. United States Amer, vol. 107, no. 3, pp. 993-998, Jan. 2010.

[20] J. Chung, W. Zheng, T. J. Hatch, and H. O. Jacobs, "Programmable reconfigurable self-assembly: Parallel heterogeneous integration of chipscale components on planar and nonplanar surfaces," J. Microelectromech. Syst., vol. 15, no. 3, pp. 457-464, Jun. 2006.

[21] T. Fukushima et al., "Reconfigured-wafer-to-wafer 3-D integration using parallel self-assembly of chips with $\mathrm{Cu}-\mathrm{SnAg}$ microbumps and a nonconductive film," IEEE Trans. Electron Devices, vol. 61, no. 2, pp. 533-539, Feb. 2014.

[22] B. Chang, I. Routa, V. Sariola, and Q. Zhou, "Self-alignment of RFID dies on four-pad patterns with water droplet for sparse selfassembly," J. Micromech. Microeng., vol. 21, no. 9, pp. 095024-095035, Sep. 2011.

[23] V. Sariola, M. Jääskeläinen, and Q. Zhou, "Hybrid microassembly combining robotics and water droplet self-alignment," IEEE Trans. Robot., vol. 26, no. 6, pp. 965-977, Dec. 2010.

[24] M. Mastrangeli, W. Ruythooren, J.-P. Celis, and C. Van Hoof, "Challenges for capillary self-assembly of microsystems," IEEE Trans. Compon. Packag. Manuf. Technol., vol. 1, no. 1, pp. 133-149, Jan. 2011.

[25] C. G. Tsai, C. M. Hsieh, and J. A. Yeh, "Self-alignment of microchips using surface tension and solid edge," Sens. Actuators A, Phys., vol. 139, no. 1 , pp. 343-349, Sep. 2007.

[26] J. S. Smith, "High density, low parasitic direct integration by fluidic self assembly (FSA)," in Proc. Electron Devices Meet., Dec. 2000, pp. 201204.

[27] C. J. Morris and B. A. Parviz, "Micro-scale metal contacts for capillary force-driven self-assembly," J. Micromech. Microeng., vol. 18, no. 1, pp. 015022-015031, Jan. 2008

[28] J. L. M. Taprogge, F. Beyeler, A. Steinecker, and B. J. Nelson, "Parallel C4 packaging of MEMS using self-alignment: Simulation and experiments," IEEE Trans. Compon. Packag. Manuf. Technol., vol. 3, no. 8, pp. 1420-1429, Aug. 2013.

[29] X. Xiong et al., "Controlled multibatch self-assembly of microdevices," J. Microelectromech. Syst., vol. 12, no. 2, pp. 117-126, Apr. 2003.

[30] M. Mastrangeli, W. Ruythooren, C. Van Hoof, and J.-P. Celis, "Characterization of interconnects resulting from capillary die-to-substrate selfassembly," in Proc. 2nd ESTC, Sep. 2008, pp. 135-140.

[31] D. A. van den Ende, R. H. L. Kusters, M. Cauwe, A. van der Waal, and J. van den Brand, "Bonding bare die LEDs on PET foils for lighting applications: Thermal design modeling and bonding experiments," in Proc. 4th ESTC, Sep. 2012, pp. 1-6.

[32] G. R. B. E. Römer et al., "Picosecond laser machining of metallic and polymer substrates for fluidic driven self-alignment," Phys. Proc., vol. 39, pp. 628-635, Nov. 2012.

[33] J. H. Brannon, J. R. Lankard, A. I. Baise, F. Burns, and J. Kaufman, "Excimer laser etching of polyimide," J. Appl. Phys., vol. 58, no. 5, pp. 2036-2043, Sep. 1985.

[34] R. Mandamparambil et al., "A comparative study of via drilling and scribing on PEN and PET substrates for flexible electronic applications using excimer and Nd:YAG laser sources," in Proc. Flexible Electron. Displays Conf. Exhibit., Feb. 2009, pp. 1-7.

[35] N. Boufercha et al., "Fluidassem-A new method of fluidic-based assembly with surface tension," in Proc. IPAS, Sep. 2008, pp. 149-159.

[36] K. A. Brakke, "The surface evolver," Exper. Math., vol. 1, no. 2, pp. 89-173, Jan. 1992.

[37] G. Arutinov, M. Mastrangeli, E. C. P. Smiths, H. F. M. Schoo, J. Brugger, and A. Dietzel, "Dynamics of capillary self-alignment for mesoscopic foil devices," Appl. Phys. Lett., vol. 102, no. 14, pp. 144101-144105, Apr. 2013

[38] K. Hermansson, U. Lindberg, B. Hok, and G. Palmskog, "Wetting properties of silicon surfaces," in Proc. Int. Conf. Solid-State Sens. Actuators, Jun. 1991, pp. 193-196. 
[39] M. Voue, M. P. Valignat, G. Oshanin, A. M. Cazabat, and J. De Coninck, "Dynamics of spreading of liquid microdroplets on substrates of increasing surface energies," Langmuir, vol. 14, no. 20, pp. 5951-5958, Sep. 1998.

[40] J. Berthier and K. Brakke, "Capillary self-assembly for 3D microelectronics," in The Physics of Microdroplets. Hoboken, NJ, USA: Wiley, 2012, pp. 185-213.

[41] K. Sato, K. Ito, S. Hata, and A. Shimokohbe, "Self-alignment of microparts using liquid surface tension-Behavior of micropart and alignment characteristics," Precision Eng., vol. 27, no. 1, pp. 42-50, Jan. 2003.

[42] B. Chang, A. Shah, I. Routa, H. Lipsanen, and Q. Zhou, "Surfacetension driven self-assembly of microchips on hydrophobic receptor sites with water using forced wetting," Appl. Phys. Lett., vol. 101, no. 11, pp. 114105-1-114105-5, 2012.

[43] C. Landesberger, E. Yacoub-George, W. Hell, and K. Bock, "Selfassembly and self-interconnection of thin RFID devices on plasmaprogrammed foil substrates," in Proc. 3rd ESTC, Sep. 2010, pp. 1-5.

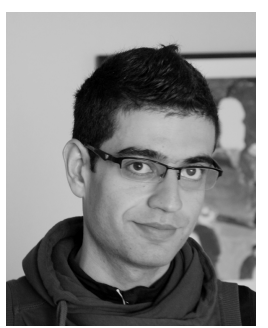

Gari Arutinov is a Ph.D. student at the Microsystems group at Eindhoven University of Technology and Marie Currie Fellow at Holst Centre/TNO. He received the B.S. and M.S. degrees (cum laude) in Applied Physics and Mathematics from Moscow Institute of Physics and Technology in 2008 and 2010 , respectively. His research interests focus on surface tension-based micromanipulation, heterogeneous system integration, self-assembly processes and flexible and stretchable electronics.

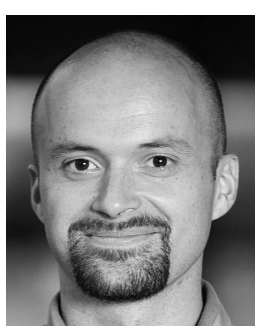

Massimo Mastrangeli received the B.S. and M.S. degrees (cum laude) in electronic engineering from University of Pisa, Pisa, Italy, in 2003 and 2005, respectively, and the Ph.D. degree in Materials Engineering from Katholieke Universiteit Leuven, Leuven, Belgium in 2010. From 2011 to 2013, he was Post-Doctoral Researcher with the Distributed Intelligent Systems and Algorithms Laboratory (DISAL) and with the Microsystems Laboratory (LMIS1) of Ecole Polytechnique Fédérale de Lausanne (EPFL), Lausanne, Switzerland. Dr. Mastrangeli is currently Senior Scientist in the department of Bio, Electro And Mechanical Systems (BEAMS) of Université Libre de Bruxelles (ULB), Bruxelles, Belgium. His current research interests include surface tension-based micromanipulation, liquid dielectrophoresis and templated nanoparticle assembly.

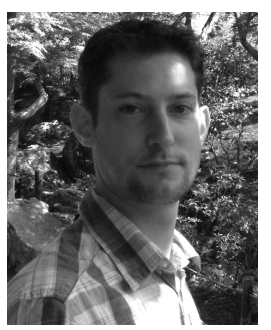

Edsger C. P. Smits received his Ph.D. under supervision of Prof. de Leeuw in 2009 from the University of Groningen in the field of organic electronics. In 2009, he started as a researcher at the Holst Centre. His current work is directed at integration solutions for plastic electronics and on the development of laser transfer printing processes. $\mathrm{He}$ is authors and co-author of over 40 peer-reviewed articles.

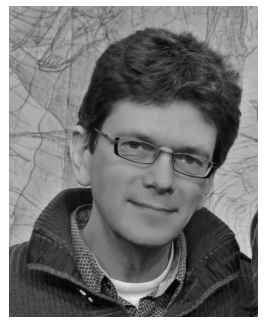

Gert van Heck is educated as an electrical engineer. From 1985 on, he has been a researcher at TNO. At the beginning of Holst Centre in 2005, he joined the group of Sensors and Actuators. His current research interests include laser processing of materials. He has (co-)authored over 50 patent applications.

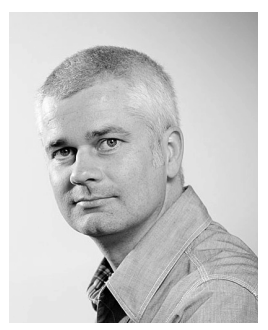

Jaap M. J. den Toonder is full professor and Chair of the Microsystems group at Eindhoven University of Technology, and is affiliated with the Institute of Complex Molecular Systems (ICMS). He studied at the Delft University of Technology and got his Master's degree in Applied Mathematics in 1991 (cum laude). He received a $\mathrm{PhD}$ degree (cum laude) from the same university in 1996. In 1995, he joined the Philips Research Laboratories in Eindhoven, The Netherlands, where he started working in the field of the mechanics of solid materials. In 2008, he became Chief Technologist, leading the R\&D program on (micro-)fluidics, and (starting in 2011) materials science and engineering. Next to his main job at Philips, Jaap den Toonder was a part-time professor at the Materials Technology group of the Eindhoven University of Technology between 2004 and 2013. His current main research interests are: microfluidics, out-ofcleanroom micro-fabrication technologies, mechanical properties of biological cells and tissues, natureinspired micro-actuators, organs on chips. Jaap den Toonder has (co-)authored over 80 scientific papers, as well as over 40 patent applications. He was a member of the Editorial Board of Lab on a Chip from 2009 to 2013

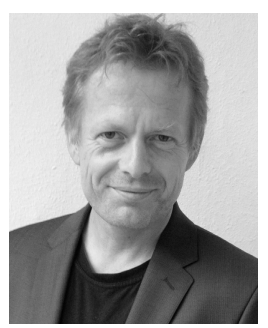

Andreas Dietzel received his diploma and $\mathrm{PhD}$ degrees in physics from the University of Göttingen (Germany) in 1986 and 1990, respectively. From 1990 to 1994, he worked in the IBM Laboratory of Surface and Structure Analysis in Boeblingen (Germany) and from 1994 to 1996 at the IBM Research Laboratory in Rueschlikon (Switzerland) in the Optoelectronics Department. In 1996, he joined the IBM Storage Technology Division in Mainz (Germany) as head of the Laboratory for Magnetic Characterization and Technology Projects. In 2003, he joined Robert Bosch $\mathrm{GmbH}$ as project manager for next generation acceleration sensors. In 2004, he was appointed full professor for the new section micro- and nano-scale engineering in the Department of Mechanical Engineering of the TU Eindhoven (The Netherlands). In the years 2007 to 2011, he also held the position of program manager for systems-in-foilintegration at the Holst Centre for shared research (TNO Eindhoven). In 2012, he was appointed professor at TU Braunschweig (Germany) and became head of the Institute of Microtechnology (IMT). His current research interests include sensors and actuators for metrological and aeronautical applications. 